\title{
Effect of Inductive Coil Geometry and Current Sheet Trajectory of a Conical Theta Pinch Pulsed Inductive Plasma Accelerator
}

\author{
Ashley K. Hallock* \\ Princeton University, Princeton, NJ, 08544 \\ Kurt A. Polzin ${ }^{\dagger}$ and Kevin W. Bonds ${ }^{\ddagger}$ \\ NASA - Marshall Space Flight Center, Huntsville, AL 35812 \\ Gregory D. Emsellem ${ }^{\S}$ \\ The Elwing Company, Wilmington, DE, 19801
}

\begin{abstract}
Results are presented demonstrating the effect of inductive coil geometry and current sheet trajectory on the exhaust velocity of propellant in conical theta pinch pulsed inductive plasma accelerators. The electromagnetic coupling between the inductive coil of the accelerator and a plasma current sheet is simulated, substituting a conical copper frustum for the plasma. The variation of system inductance as a function of plasma position is obtained by displacing the simulated current sheet from the coil while measuring the total inductance of the coil. Four coils of differing geometries were employed, and the total inductance of each coil was measured as a function of the axial displacement of two separate copper frusta both having the same cone angle and length as the coil but with one compressed to a smaller size relative to the coil. The measured relationship between total coil inductance and current sheet position closes a dynamical circuit model that is used to calculate the resulting current sheet velocity for various coil and current sheet configurations. The results of this model, which neglects the pinching contribution to thrust, radial propellant confinement, and plume divergence, indicate that in a conical theta pinch geometry current sheet pinching is detrimental to thruster performance, reducing the kinetic energy of the exhausting propellant by up to $50 \%$ (at the upper bound for the parameter range of the study). The decrease in exhaust velocity was larger for coils and simulated current sheets of smaller half cone angles. An upper bound for the pinching contribution to thrust is estimated for typical operating parameters. Measurements of coil inductance for three different current sheet pinching conditions are used to estimate the magnetic pressure as a function of current sheet radial compression. The gas-dynamic contribution to axial acceleration is also estimated and shown to not compensate for the decrease in axial electromagnetic acceleration that accompanies the radial compression of the plasma in conical theta pinches.
\end{abstract}

\section{Nomenclature}

$\begin{array}{llll}\bar{A} & \text { cross sectional area }\left(\mathrm{m}^{2}\right) & M & \text { mutual inductance, }(\mathrm{H}) \\ \alpha & \text { half cone angle (degrees) } & m & \text { current sheet mass, }(\mathrm{kg}) \\ C & \text { capacitance (F) } & P, P_{1}, P_{2} & \text { gas-dynamic, initial, final pressure }(\mathrm{Pa}) \\ F_{j}, F_{r} & \text { Force }(\mathrm{N}) & R_{e}, R_{p} & \text { external circuit, plasma resistance }(\Omega) \\ I_{1}, I_{2}, I_{r m s} & \text { coil, plasma, RMS current }(\mathrm{A}) & R, r & \text { major, minor radius }(\mathrm{m})\end{array}$

\footnotetext{
${ }^{*}$ Graduate Student, Mechanical and Aerospace Engineering Department, E-Quad Olden St. Princeton, NJ 08544, Student Member AIAA.

${ }^{\dagger}$ Propulsion Research Engineer, Propulsion Research and Technology Applications Branch,Propulsion Systems Department, Senior Member AIAA.

$¥$ Intern presently undergraduate student at Middle Tennessee State University, Student Member AIAA.

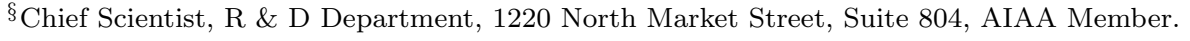




$\begin{array}{llll}k & \text { transformer coupling coefficient } & \rho_{A} & \text { linear mass distribution }(\mathrm{kg} / \mathrm{m}) \\ L_{0}, L_{C} & \text { parasitic, accessible inductance }(\mathrm{H}) & V & \text { voltage }(\mathrm{V}) \\ L^{*} & \text { nondimensional inductance } & \mathbb{V} & \text { volume }\left(\mathrm{m}^{3}\right) \\ l_{\text {coil }} & \text { coil length }(\mathrm{m}) & v_{p}, v_{u p}, v_{z} & \text { pinched, unpinched, axial velocity }(\mathrm{m} / \mathrm{s})\end{array}$

\section{Introduction}

$\mathrm{T}_{\mathrm{T}}^{\mathrm{T}}$ is desirable to extend the lifetime and increase the reliability of an in-space propulsion system as much 1 as possible since that maintenance or replacement of that system becomes particularly challenging once it has been placed into orbit. Reducing the size and mass of the propulsion system, including the propellant required to complete a mission can permit an increase in the amount of payload as a percentage of total vehicle mass. The high values of specific impulse associated with electric propulsion (EP) reduces the amount of propellant needed for a given mission relative to other conventional propulsion systems.

Pulsed inductive plasma thrusters[1-3] are spacecraft propulsion devices in which electrical energy is capacitively stored and then discharged through an inductive coil. The thruster is electrodeless, with a timevarying current in the coil interacting with a plasma covering the face of the coil to induce a plasma current. Propellant is accelerated and expelled at a high exhaust velocity $(\mathcal{O}(10-100 \mathrm{~km} / \mathrm{s}))$ by the Lorentz body force arising from the interaction of the magnetic field and the induced plasma current.

Thrusters of this type possess many demonstrated and potential benefits that make them worthy of continued investigation. The electrodeless nature of these thrusters eliminates the lifetime and contamination issues associated with electrode erosion in conventional electric thrusters. Also, a wider variety of propellants are available for use when compatibility with metallic electrodes in no longer an issue. Pulsed inductive accelerators have been successfully operated using propellants like ammonia, hydrazine, and $\mathrm{CO}_{2}$, and there is no fundamental reason why they would not operate on other propellants like $\mathrm{H}_{2} \mathrm{O}$. It is well-known that pulsed accelerators can maintain constant specific impulse $I_{\mathrm{sp}}$ and thrust efficiency over a wide range of input power levels by adjusting the pulse rate to maintain a constant discharge energy per unit pulse. It has also been demonstrated that a pulsed inductive thruster operating in or near the regime of optimum dynamic impedance matching can operate at a relatively constant thrust efficiency over a wide range of $I_{\mathrm{sp}}$ values. Thrusters in this class have operated at high energy per pulse, and by increasing the pulse rate they offer the potential to process very high levels of power using a single thruster.
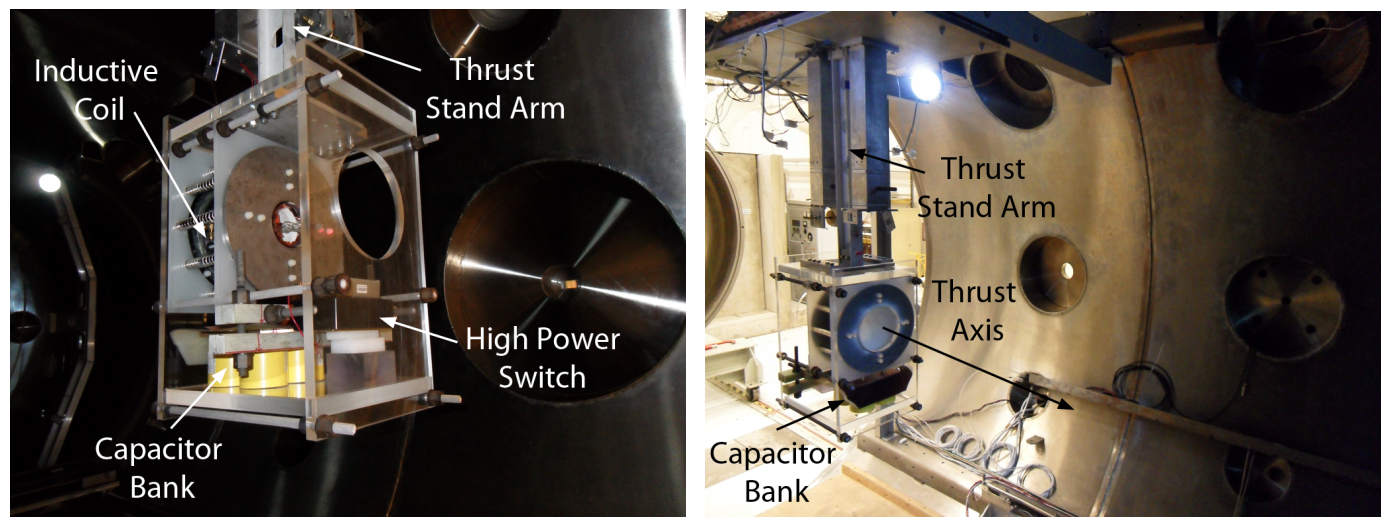

Figure 1. Photographs of the MAD-IPA mounted on the VAHPER [4] thrust stand.

The capacitors in some inductive accelerators, like the Pulsed Inductive Thruster (PIT) [1-3], must be charged to high voltages so that the induced fields can first ionize the propellant. One way to alleviate this high voltage requirement is to partially ionize the propellant in front of the inductive coil such that the induced electric field only needs to perform an under-voltage breakdown of already partially-ionized propellant[5], allowing for a lower initial charge voltage on the capacitors. The use of preionization in pulsed inductive devices is ubiquitous throughout the literature with a wide range of applications including plasma fusion and spacecraft propulsion. For example, preionization has been successfully employed by striking a glow discharge between two electrodes [6-8], sending a separate lower-energy pulse through an inductive 
coil[9-11], and creating a radio frequency plasma [12,13]. Not all of the propellant must be preionized because only the preionized propellant within a few characteristic length scales of the inductive coil has any significant interaction with fields induced by the high-current pulse.

The Microwave Assisted Discharge Inductive Plasma Accelerator (MAD-IPA) [14,15], shown in Fig. 1, employs an electron cyclotron resonance to partially ionize propellant in a thin region along the inner surface of the inductive coil. Current sheet formation at this location initially presents the minimum inductance to the capacitor bank, which is advantageous in that it allows for the maximum potential conversion of electromagnetic field energy to work accelerating the propellant. Current sheet formation further from the coil represents a loss in achievable electromagnetic acceleration, which is represented as a greater initial inductance presented to the capacitor bank. A photograph of the microwave-driven preionization stage of the MAD-IPA in operation is shown in Fig. 2.

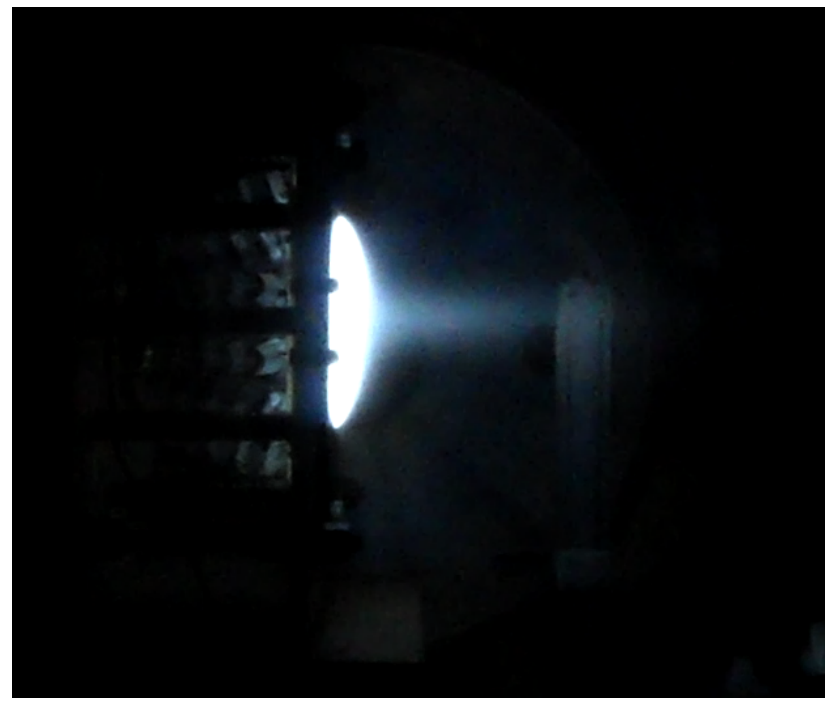

Figure 2. A photograph of the MAD-IPA preionization stage operating on air at 2.7 Pascal (20 mtorr).

In this paper, we present a study of the effect of inductive coil geometry and current sheet trajectory on the inductive electromagnetic coupling efficiency of the MAD-IPA and other conical theta pinch thrusters that rely on current sheet acceleration of the plasma and operate at a propellant mass per pulse of between $1-10 \mathrm{mg}$. This work and the results presently do not encompass devices that employ compact toroid plasmas or other very high gas density discharges. Experimental data are used to close a well-known model of thruster performance to bound the current sheet exhaust velocity as a function of coil geometry and radial current sheet compression. The rest of this paper describes the lumped-element circuit model used to calculate current sheet acceleration, an experiment that yielded semi-empirical relations for various coil geometries and current sheet trajectories to close the circuit model, the results of the model calculations, and a discussion of the trends thruster performance.

\section{Inductive Accelerator Modeling}

Pulsed inductive thrusters are commonly studied with the use of a semi-empirical circuit model coupled to a one dimensional momentum equation [2]. This circuit is shown in Fig. II, where $I_{1}$ is the current flowing in the driving circuit, $I_{2}$ is the current flowing in the plasma current sheet, $C$ is the capacitance of the capacitor, $M$ is the mutual inductance between the driving coil and the current sheet, $L_{0}$ is the initial (parasitic) inductance, $L_{C}$ is the accessible coil inductance, $R_{p}$ is the resistance of the plasma, and $R_{e}$ is the resistance in the driving circuit.

The equivalent circuit shown in Fig. 4 can be drawn and a set of equations can be derived to model thruster performance in terms of these electrical parameters. From this circuit diagram, where $V_{0}$ is the initial voltage on the capacitor and $V$ is the voltage on the capacitor as a function of time, the following equations follow from the application of Kirchoff's law: 


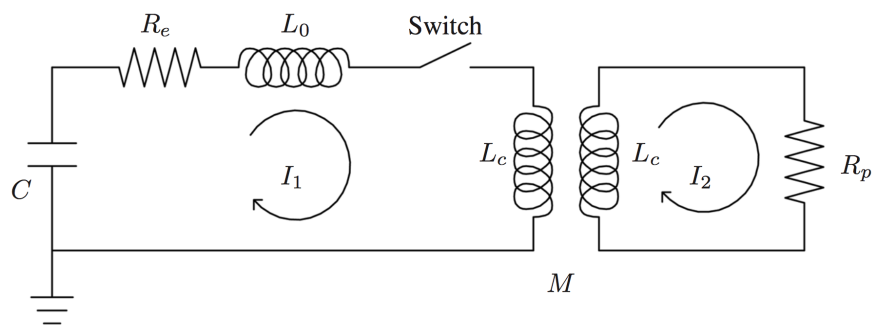

Figure 3. Lumped element circuit model of a pulsed inductive thruster inductively coupled to a plasma (taken from Ref. [2]).

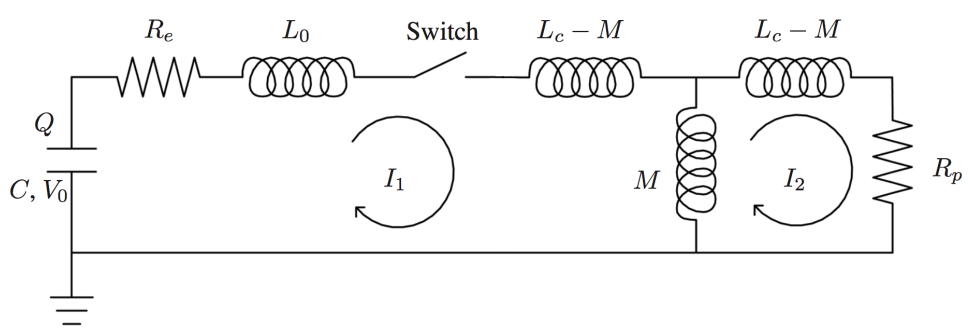

Figure 4. Equivalent circuit of a pulsed inductive thruster inductively coupled to a plasma used to derive a set of governing equations to model thruster performance.

$$
\begin{gathered}
\frac{d I_{1}}{d t}=\frac{L_{C} V-L_{C} R_{e} I_{1}-M R_{p} I_{2}+\left(L_{C} I_{2}+M I_{1}\right) \frac{d M}{d t}}{L_{C}\left(L_{0}+L_{C}\right)-M^{2}} \\
\frac{d I_{2}}{d t}=\frac{M \frac{d I_{1}}{d t}+I_{1} \frac{d M}{d t}-R_{p} I_{2}}{L_{C}} \\
\frac{d V}{d t}=\frac{-I_{1}}{C}
\end{gathered}
$$

The equation of motion for the current sheet can be written using Newton's second law with the force acting on the current sheet arising from the magnetic pressure between the driving coil and the current sheet propellant mass in the sheet accumulating according to the snowplow model.

$$
\frac{d v_{z}}{d t}=\left[\frac{L_{C} I_{1}^{2}}{2 z_{0}} \exp \left(-\frac{z}{z_{0}}\right)-\rho_{A}(z) v_{z}^{2}\right] / m(z)
$$

where $z$ is the axial displacement of the current sheet from the driving coil, $m(z)$ is the total propellant mass in the current sheet, $\rho_{A}(z)$ is the linear mass density distribution, and $v_{z}$ is the axial current sheet velocity.

$$
\frac{d z}{d t}=v_{z}
$$

For this study, the current sheet was modeled as a slug mass $\left(\rho_{A}(z)=0, m_{0}=m_{b i t}\right)$. The snowplow accumulated mass in the sheet is then given by

$$
m(t)=\int_{0}^{t} \rho_{A} v_{z} d t^{\prime}+m_{0}
$$

Adding the lumped inductive elements shown in Fig. 4 gives the total inductance,

$$
L_{t o t}=L_{0}+L_{C}-\frac{M^{2}}{L_{C}} .
$$


It can be seen from this equation that the total inductance changes as a function of time due to the time changing mutual inductance, which varies due to the movement of the current sheet. An expression for the mutual inductance as a function of current sheet axial position has been empirically determined [2] for a half-cone angle of $90^{\circ}$ (i.e. flat coil):

$$
L_{t o t}(z)=L_{0}+L_{C}\left(1-\exp \left(-z / z_{0}\right)\right)
$$

where $z_{0}$ is the decoupling distance. This expression is set equal to the previous expression for total inductance (Eq. 7) and solved for the mutual inductance as a function of the axial separation distance between the driving coil and the current sheet:

$$
M=L_{C} \exp \left(-z / 2 z_{0}\right)
$$

of which the time derivative is,

$$
\frac{d M}{d t}=-\frac{L_{C}}{2 z_{0}} \exp \left(-z / 2 z_{0}\right) \frac{d z}{d t}
$$

closing the set of six first-order ODEs, consisting of Eqs. 1, 2, 3, 4, 5, and 10, that can be readily solved numerically. Of these six governing equations only Eq. 10 must be empirically found based upon the inductive coil geometry. While Eq. 8 was developed for a planar coil geometry, it has been found to accurately represent the axial inductive coupling behavior of ring-shaped and conical geometries as well $[16,17]$.

We nondimensionalize Eq. 8 by $L_{C}$ to produce a general relation for the change in total measured inductance as a function of current sheet axial position:

$$
L_{t o t} / L_{C}=L_{0} / L_{C}+\left(1-\exp \left(-z / z_{0}\right)\right)
$$

The first term on the right hand side of Eq. 11 represents a constant parasitic inductance inaccessible for current sheet acceleration. The second term represents the total accessible inductance potentially convertible to current sheet acceleration. It is this second term that isolates the effect of coil geometry on current sheet acceleration, and we label this value $L^{\diamond}$ :

$$
L^{\diamond}=\frac{L_{t o t}-L_{0}}{L_{C}}=1-\exp \left(-z / z_{0}\right)
$$

\section{Experiment}

If the value of $d M / d t$ were known as a function of coil geometry at each axial location of the current sheet, the model from section II could be solved numerically to calculate the effect of coil geometry on thruster performance. To estimate this relation, we constructed inductive coils of various geometries and measured the total inductance of these coils as a function of the displacement of a copper frustum that simulates the presence of a current sheet. We assume that the current sheet geometry will mirror the coil geometry that formed it, and constructed two simulated current sheets for each coili geometry in the form of copper frusta. One frustum fits tightly against the inner surface of the coil while the second has a different minor radius $r_{c s}$ to simulate radial compression (or pinching) of the current sheet. Pinching motion is assumed to leave the half cone angle $\alpha$ and coil length $l_{\text {coil }}$ unchanged where $l_{\text {coil }}$ is defined as:

$$
l_{\text {coil }}=\left(R_{\text {coil }}-r_{\text {coil }}\right) / \tan (\alpha) .
$$

Copper frusta were constructed from flat copper sheets whose thickness (0.062 inches) captures $99.9 \%$ of the energy of an impinging electromagnetic field. The copper frusta were electrically isolated and fitted with wooden conic sections that hold a fiberglass bolt on the centerline. A schematic of the experimental setup is shown in Fig. 5 and a photograph of the inductive coils and their associated copper frusta are shown in Fig. 6 .

The inductive coils were constructed from 0.008 " thick copper and 0.02" thick mylar. Four different inductive coil geometries were studied, and for each inductive coil geometry two current sheet geometries were studied representing two different current sheet trajectories. It is assumed that the current sheet geometries mirror those of the inductive coils that would have created them. These geometries are described in Tables $1 \& 2$ (the "P" at the end of those references associated with radially compressed current sheet geometries stands for "pinched"). 

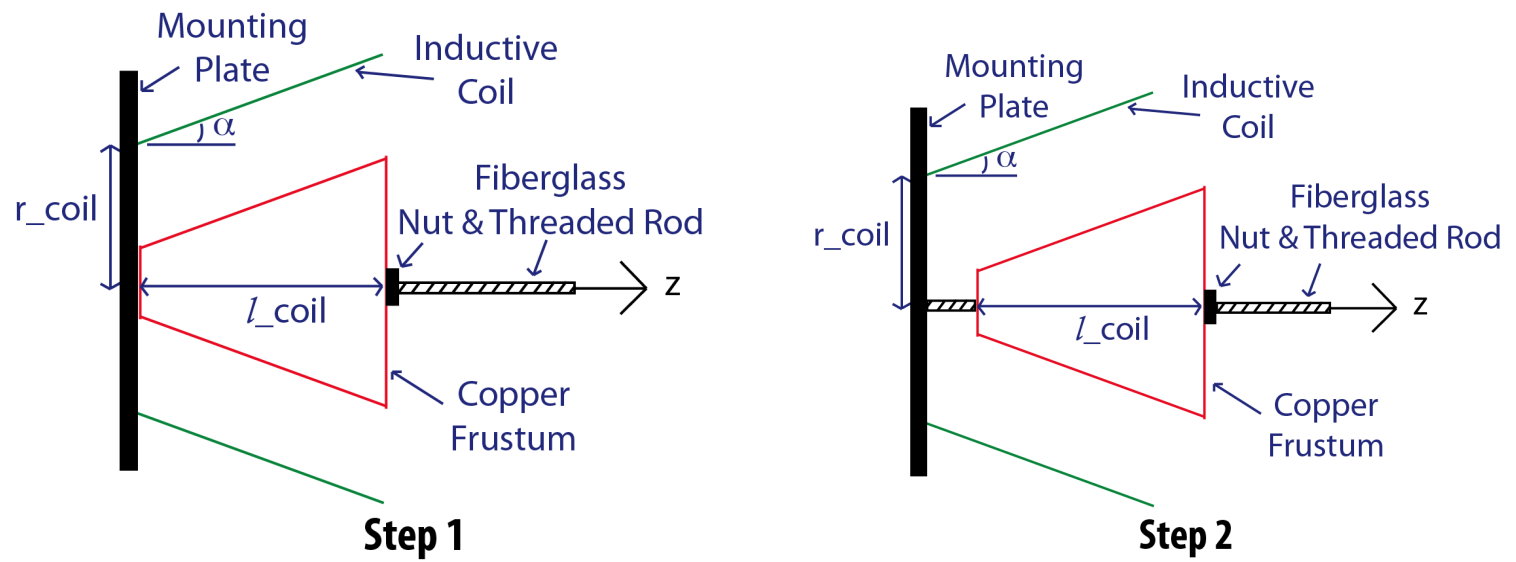

Figure 5. Schematic of controlled copper frustum displacement from the inductive coil.

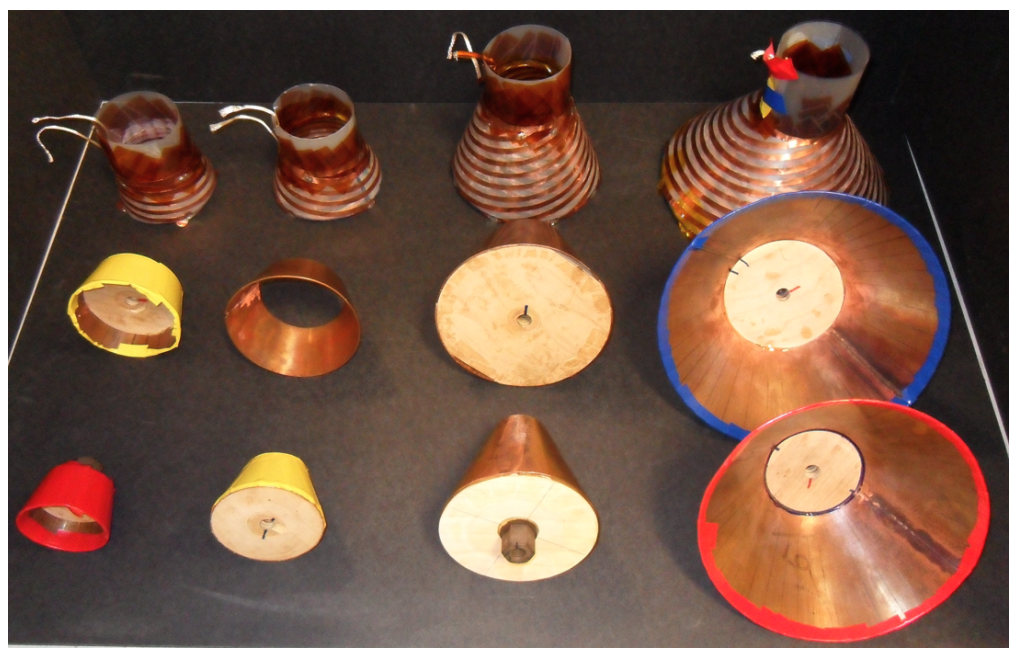

Figure 6. Coils and copper conic frusta used in the experiment.

The total inductance $\left(L_{t o t}\right)$ was measured at 84 locations using an Agilent $4285 \mathrm{~A}$ precision LCR meter and is shown in Fig. 7 as a function of axial copper frustum displacement. We calculate values of $L_{0}, L_{C}$, and $z_{0}$ for each coil geometry by fitting these data to Eq. 8. Values for $L_{C}$ and $z_{0}$ are shown in Table 3 for the four different coil geometries studied. As $L_{0}$ represents inaccessible inductance, we associate this value with the parasitic inductance of the driving circuit, and assume it is not significantly affected by coil geometry.

The coil with the lowest accessible inductance $\left(L_{C}\right)$, 20L, is taken as a baseline to which the other geometries can be compared. The larger half cone angle of coil 38 allows the field created by a current pulse through its conductive traces to develop in a larger volume and leads to a higher total flux for the same current. The shorter inductive coils have a higher accessible inductance for two reasons. The shorter length also allows the field to develop in a larger volume, leading to a higher total flux. A secondary reason relates to the construction process. In shortening the length, we attempted to maintain the number of turns, but in so doing, decreased the pitch angle of the traces such that the azimuthal component of the current in the traces was increased. Since all other components of the current cancel by design of the inductive coil trace pattern, the field is stronger for a given applied current in the shorter coil, leading to a higher accessible inductance.

The loss of accessible inductance incurred by the pinched current sheets relative to the unpinched current sheets, shown in Table 4, was calculated by taking the difference between $L_{C}$ at $z=0$ for the pinched and unpinched current sheets. This additional inductance can be seen in Fig. 7 as a higher value for inductance 


\begin{tabular}{|l|c|c|c|}
\hline reference & $\alpha$ (degrees) & $l_{\text {coil }}(\mathrm{cm})$ & $r_{\text {coil }}(\mathrm{cm})$ \\
\hline 12 & 12 & 4.3 & 4 \\
$20 \mathrm{~S}$ & 20 & 5 & 4 \\
$20 \mathrm{~L}$ & 20 & 10 & 4 \\
38 & 38 & 10 & 4 \\
\hline
\end{tabular}

Table 1. Inductive coil geometries studied.

\begin{tabular}{|l|c|c|c|}
\hline reference & $\alpha$ (degrees) & $l_{\text {coil }}(\mathrm{cm})$ & $r_{c s}(\mathrm{~cm})$ \\
\hline CS12 & 12 & 4.3 & 3.9 \\
CS12P & 12 & 4.3 & 2.5 \\
CS20S & 20 & 5 & 3.9 \\
CS20SP & 20 & 5 & 2.5 \\
CS20L & 20 & 10 & 3.9 \\
CS20LP & 20 & 10 & 2.5 \\
CS38 & 38 & 10 & 3.9 \\
CS38P & 38 & 10 & 2.5 \\
\hline
\end{tabular}

Table 2. Current sheet geometries studied.

\begin{tabular}{|l|c|c|}
\hline Current Sheet & $L_{C}(\mathrm{nH})$ & $z_{0}(\mathrm{~cm})$ \\
\hline CS12 & 564 & 2.6 \\
CS20S & 624 & 2.6 \\
CS20L & 450 & 4.0 \\
CS38 & 558 & 3.6 \\
\hline
\end{tabular}

Table 3. Fit parameters for various unpinched current sheet geometries. 

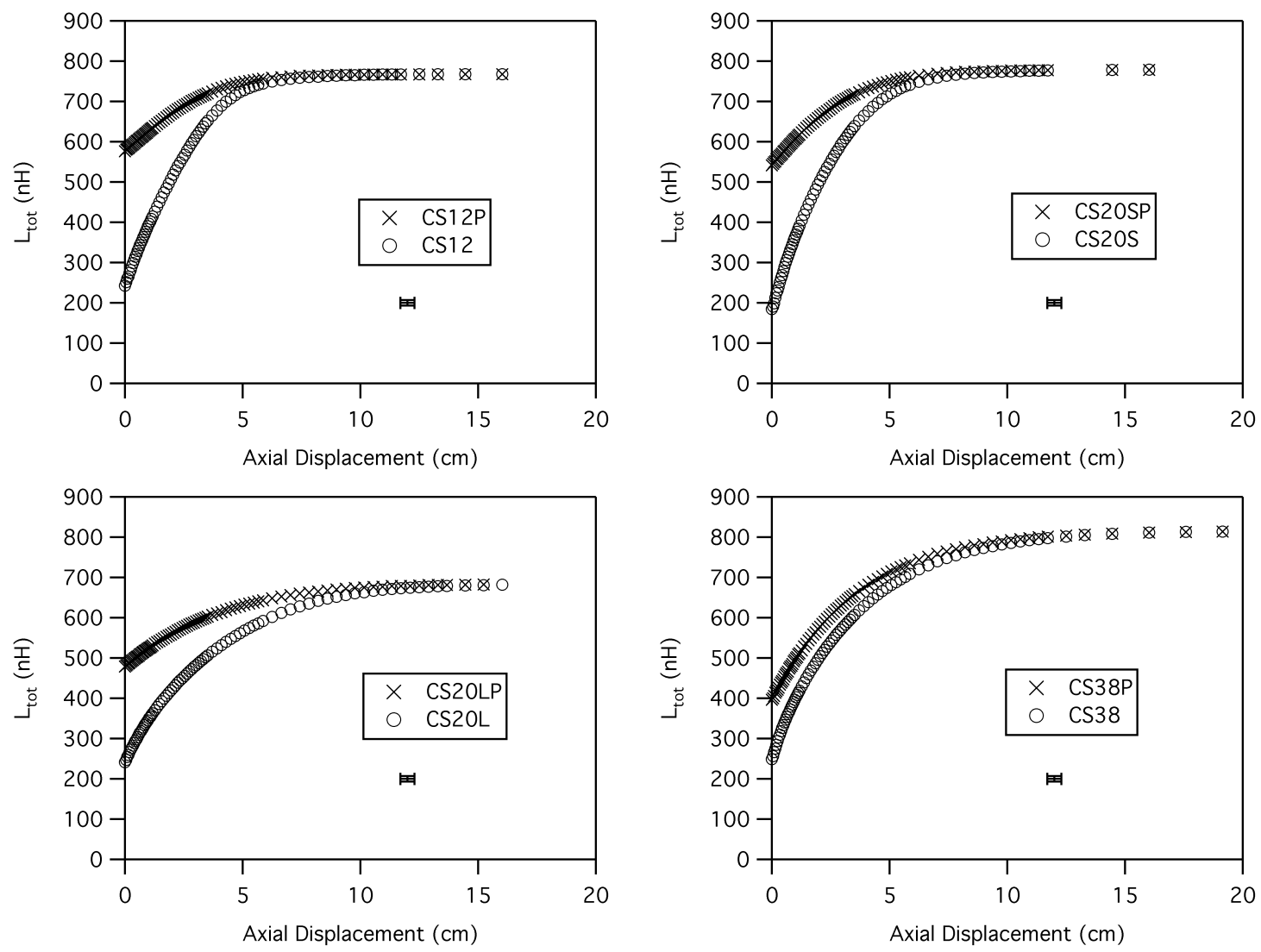

Figure 7. Experimentally measured total inductance for various inductive coil geometries as a function of current sheet displacement with a typical error bar shown. Pinched current sheet trajectories are represented as crosses and unpinched current sheets are represented as circles.

at $z=0$ for the pinched current sheets. From these data we conclude that pinched current sheets show weaker initial coupling to the inductive coil.

\begin{tabular}{|l|c|c|}
\hline Current Sheet & $L_{0}(\mathrm{nH})$ & $z_{0}(\mathrm{~cm})$ \\
\hline CS12P & 445 & 2.6 \\
CS20SP & 466 & 2.6 \\
CS20LP & 344 & 4.0 \\
CS38P & 247 & 3.6 \\
\hline
\end{tabular}

Table 4. Additional parasitic inductance values of various pinched current sheet geometries.

To isolate the effect of coil geometry from the influence of the driving circuit, we calculated $L^{\diamond}$ for each axial location by removing the value for $L_{0}$ from each data point and non-dimensionalizing to $L_{C}$. The results are shown in Fig. 8 along with a plot of Eq. 12 (shown in red). Though none of the coil geometries studied resembled that of the PIT MkV coil, the data fit well.

The similarity of the nondimensionalized inductance measurements for each geometry suggests that for any given current sheet geometry within the range of parameters studied here, unique values for the accessible inductance of the driving coil, $L_{C}$, and the decoupling distance $z_{0}$ can be determined using this method. The fact that all data collapse when normalized to these variables suggests that the accessible inductance decreases in the same sense as a percentage of the initial $L_{C}$ for all four coil geometries as the current sheet displaces axially from the driving coil. Even though current sheet pinching causes a decrease in the initial accessible inductance (or increase in the initial parasitic inductance), the accessible inductance for both pinched and unpinched current sheets decreases in the same sense as a percentage of the initial value as the 


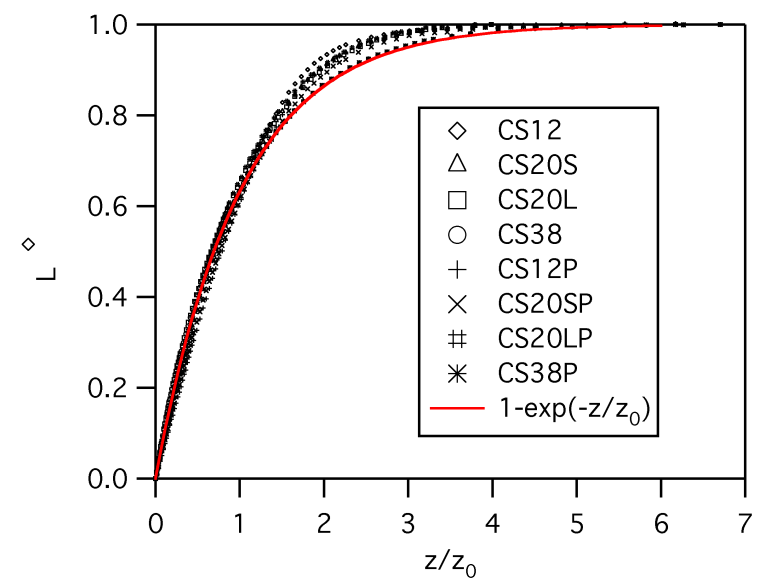

Figure 8. Plot of nondimensionalized inductance $L^{\diamond}$ vs. nondimensionalized current sheet displacement.

current sheet decouples from the coil.

\section{Discussion}

\section{A. Current Sheet Trajectory and Coil Geometry}

Experimentally determined values for $L_{0}, L_{C}$ and $z_{0}$ were inserted into Eq. 8 to model thruster performance for each current sheet trajectory for each inductive coil geometry. A constant value of parasitic inductance of $100 \mathrm{nH}$ is assumed for each case. For unpinched current sheets, this is equal to the total initial inductance of the circuit, while the additional parasitic inductance listed in Table 4 is added to the external inductance to yield a greater initial inductance for pinched current sheets. The model predicts the velocity of the current sheet as a function of time. These predictions are presented in Figs. $9 \& 10$, with the former isolating the effect of $l_{\text {coil }}$ and the latter focusing on the effects of $\alpha$ and $r_{c s}$ where $r_{c s}$ simulates current sheet pinching.

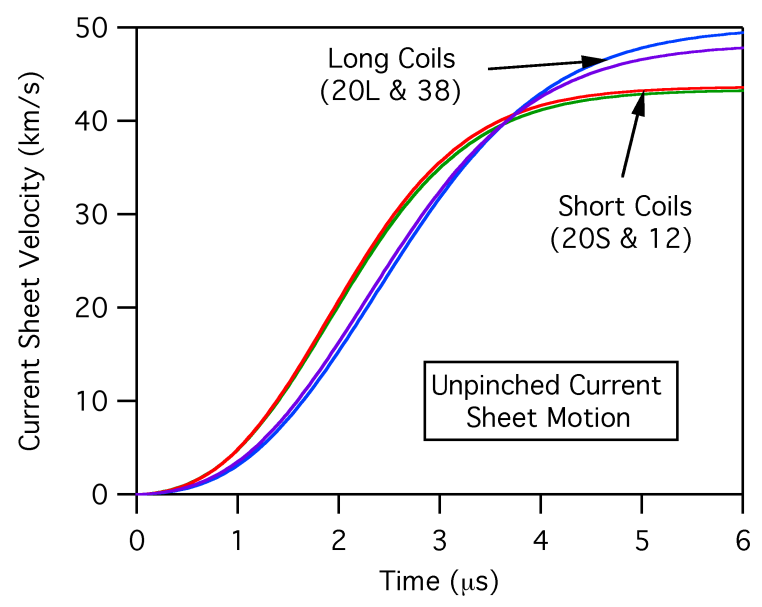

Figure 9. Current sheet velocities versus time where current sheets for longer inductive coils (20L and 38) have a more gradual acceleration that lasts longer relative to shorter inductive coils (12 and $20 \mathrm{~S})$.

The results plotted in Fig. 9 show that those axially translating current sheets created in the longer coil/current sheet combinations experience a less intense acceleration process over a longer time relative to the shorter coils, ultimately leading to a higher final exhaust velocity than in the shorter coil/current cases. This result implies that the inductance changes more rapidly for the short coils, but that the current sheet is electromagnetically coupled to the acceleration coil over a greater distance for the longer coils, leading to a higher exhaust velocity. The observed trend is preserved for the longer and shorter pinched current sheets. 

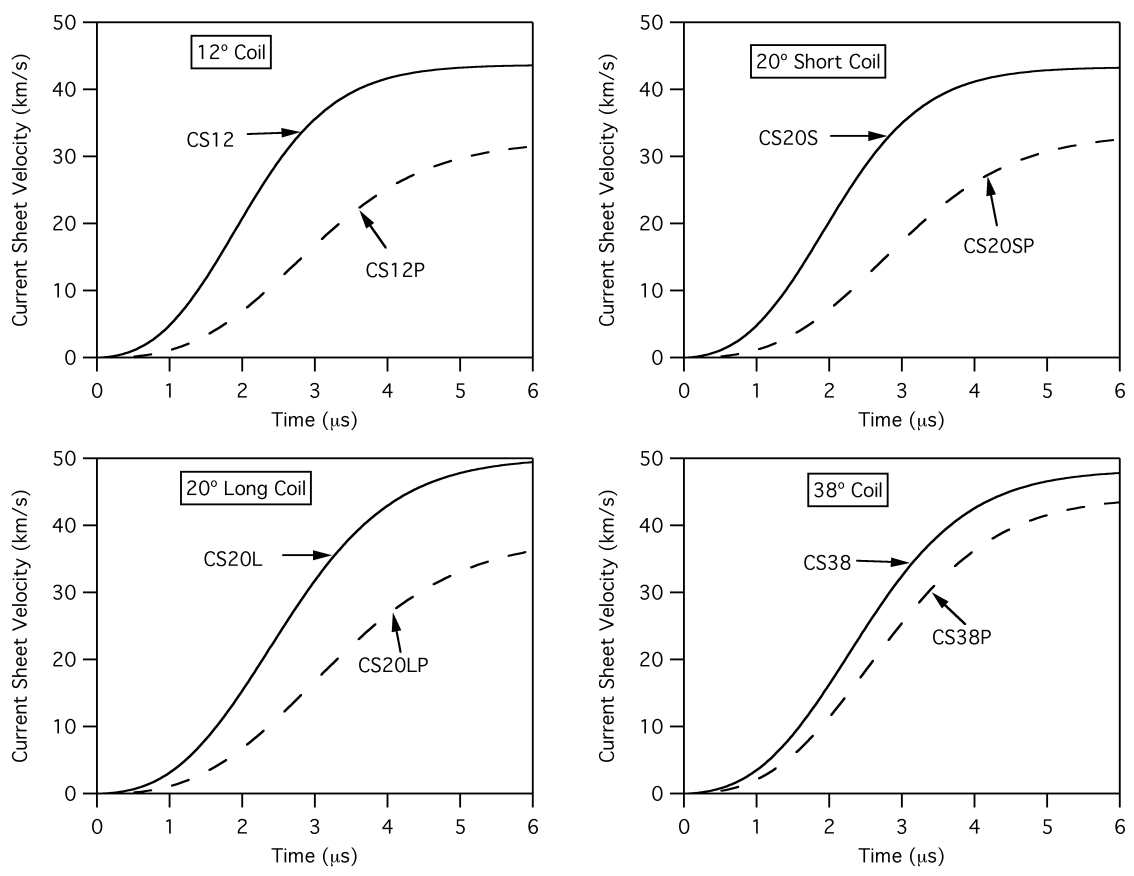

Figure 10. Results of axial plasma acceleration modeling for different inductive coil geometries with Left: un-pinched current sheets and Right: pinched current sheets.

When comparing the model results for the velocity of unpinched and pinched current sheets in Fig. 10, it appears that when the current sheet is pinched it decouples from the driving coil faster, implying a decrease in the achievable stroke length or, equivalently, an increase in the initial inductance presented to the driving circuit. Stated differently, a current sheet that experiences more pinching results in a reduction in the electromagnetic field energy available to accelerate the plasma. The final axial exhaust velocities attained by all current sheet geometries studied are shown in Table 5, along with the percentage loss of exhaust velocity and directed kinetic energy resulting from radial pinching of the current sheet.

\begin{tabular}{|l|c|l|c|c|c|}
\hline Unpinched Cases & $v_{z}(\mathrm{~km} / \mathrm{s})$ & Pinched Cases & $v_{z}(\mathrm{~km} / \mathrm{s})$ & velocity loss $\frac{\Delta v}{v_{z \max }}$ & KE loss \\
\hline CS12 & 44 & CS12P & 31 & $30 \%$ & $50 \%$ \\
CS20S & 43 & CS20SP & 33 & $23 \%$ & $41 \%$ \\
CS20L & 49 & CS20LP & 36 & $27 \%$ & $46 \%$ \\
CS38 & 48 & CS38P & 43 & $10 \%$ & $20 \%$ \\
\hline
\end{tabular}

Table 5. Final exhaust velocities for various current sheet geometries.

As the half cone angle of an inductive coil is increased the accelerator exhibits lower susceptibility to performance loss due to radial current sheet displacement. The explanation for this property lies in the change in volume available for a magnetic field to occupy in each conical inductive coil/current sheet configuration relative to the initial available volume. For efficient thruster operation, the initial inductance presented to the circuit by the coil should be minimized, or stated another way the initial volume occupied by the field generated by the coil should be minimized. This is accomplished when the current sheet forms as close to the driving coil as possible because the field from the current sheet and the coil are summed between the coil and the current sheet and mostly cancel within the rest of the domain. A radial displacement of the current sheet increases the available volume between coil and current sheet less for coils of larger $\alpha$. The decrease in the volume enclosed by the current sheet $\Delta \mathbb{V}$ as a fraction of the initial volume enclosed by the current sheet $\mathbb{V}_{0}$ is shown in Fig. 11 for the four coil geometries studied. This change in volume is representative of a loss of volume capable of shielding field generation at $z=z_{0}$.

The model suggests that radial displacement of the current sheet is purely detrimental to its final exhaust 


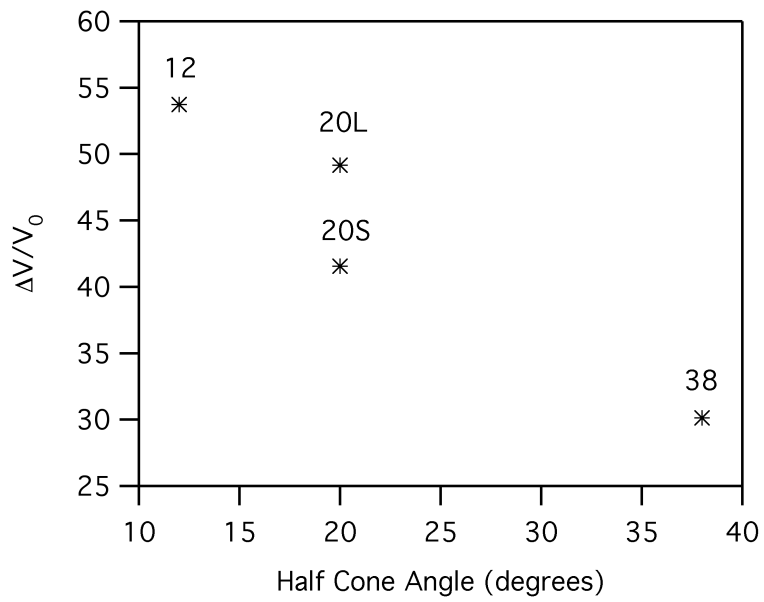

Figure 11. Coils with a larger half cone angle experience a smaller loss of volume in which magnetic field is shielded during a current pulse.

velocity, however the model only takes into account electromagnetic acceleration of the plasma current sheet. Radial displacement may increase propellant utilization efficiency or increase overall thruster efficiency by decreasing plume divergence. It may also contribute directly to plasma acceleration in the form of a gas-dynamic pressure imbalance brought about by compression (or pinching) of the current sheet and the entrained propellant. We proceed with a discussion aimed at estimating an upper bound on the pinching contribution to axial thrust in these thrusters.

\section{B. Potential Gas-dynamic Pinching Contribution to Thrust}
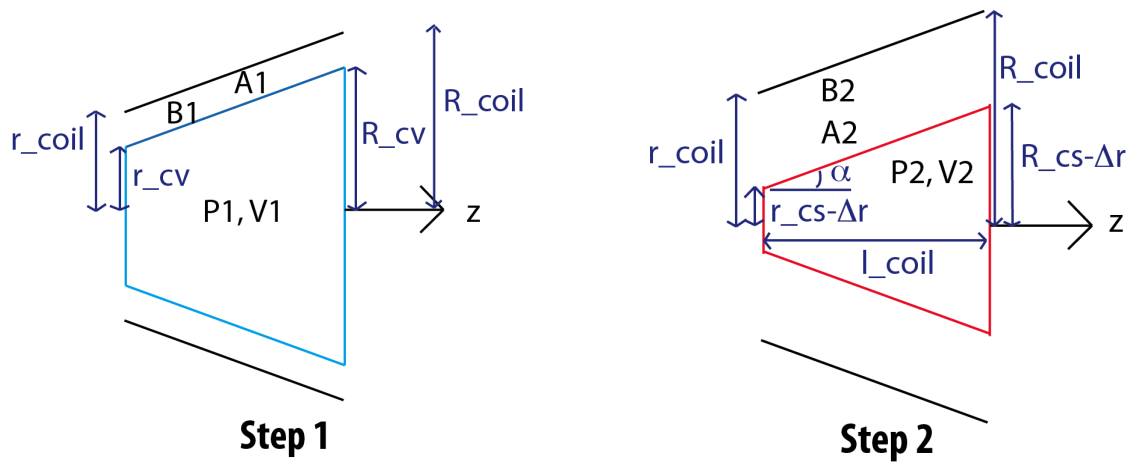

Figure 12. Side view of coil and current sheet geometry Left: Initial conditions and Right: after pinching

To estimate an upper bound on the pinching contribution to thrust that will arise through radial plasma compression, we make assumptions that will overestimate the achievable gas-dynamic propellant pressure. These assumptions are:

1. The number of particles in the control volume (shown in blue and red in Fig. 12) remains constant.

2. Gas-dynamic compression of the current sheet is isentropic.

3. The control volume geometry mirrors the coil geometry, with the pinched current sheet displaced radially where only the minor and major radii $\left(r_{c s}\right.$ and $R_{c s}$ respectively) of the current sheet volume change while the length $l_{\text {coil }}$, half cone angle $\alpha$ and axial $z$ location remain constant.

For isentropic compression we write:

$$
P \mathbb{V}^{\gamma}=\text { constant }
$$


where $P$ is the pressure, $V$ is volume, and $\gamma$ is the ratio of specific heats (taken as 1.4 for the present case). From the uncompressed state 1 to the pinched state 2 the pressure change can be written as

$$
P_{2}=\left(\frac{\mathbb{V}_{1}}{\mathbb{V}_{2}}\right)^{\gamma} P_{1}
$$

The initial volume $V_{1}$ is

$$
\mathbb{V}_{1}=\frac{\pi l_{c o i l}}{3}\left(R_{c s}^{2}+R_{c s} r_{c s}+r_{c s}^{2}\right)
$$

and under radial compression of $\Delta r$ the final volume is:

$$
\mathbb{V}_{2}=\frac{\pi l_{c o i l}}{3}\left(\left(R_{c s}-\Delta r\right)^{2}+\left(R_{c s}-\Delta r\right)\left(r_{c s}-\Delta r\right)+\left(r_{c s}-\Delta r\right)^{2}\right),
$$

We proceed with a rough estimate of the magnetic pressure acting on the plasma. In Fig. 7 the inductance presented to the thruster is seen to increase as the conductor pinches inward. As a very rough estimate, we assume for this zeroth-order analysis that the total inductance has a radial variation given as

$$
L_{\text {tot }}(\bar{r})=L_{0}+L_{C}\left(1-\left(\frac{\bar{r}}{\overline{r_{\text {coil }}}}\right)^{N}\right)
$$

where $\bar{r}$ is the average radial position of the current sheet

$$
\bar{r}=\frac{\left(R_{c s}-\Delta r\right)+\left(r_{c s}-\Delta r\right)}{2},
$$

$\overline{r_{\text {coil }}}$ is the average radial location of the coil

$$
\overline{r_{\text {coil }}}=\frac{R_{\text {coil }}+r_{\text {coil }}}{2},
$$

and $N$ is a parameter that depends on coil geometry. For coils 12, 20S, and 20L, values for $N$ are chosen to fit the inductance data in Section III as well as possible. For coil 38, a fit of the small set of data available leads to a value of $N<2$, which is unphysical because it would imply that the magnetic pressure on the current sheet increases as the sheet compresses. This may imply that the form assumed for $\bar{r}$ is incorrect or needs refinement, or it could simply mean that the present data set for coil 38 is too sparse to accurately capture the value of $N$. In either case, more data showing the variation of $L_{t o t}(\bar{r})$ should help reveal the full nature of and correct form of Eq. 13.

The inductive force on the current sheet in the $j$-th direction is [2]

$$
F_{j}=\frac{I_{1}^{2}}{2} \frac{d L}{d x_{j}} .
$$

Force in the $z$-direction yields axial acceleration. Using Eq. 13 the radial force can be written as

$$
F_{r}=-N I_{1}^{2} L_{C} \frac{\bar{r}^{N-1}}{{\overline{R_{\text {coil }}}}^{N}}
$$

where values for $N$ are shown in Table 6

\begin{tabular}{|l|c|}
\hline reference & $N$ \\
\hline CS12 & 3.5 \\
CS20S & 3.2 \\
CS20L & 3.2 \\
CS38 & 2 \\
\hline
\end{tabular}

Table 6. Values for the parameter $N$ that estimates the dependence of $L_{t o t}(\bar{r})$ on $\alpha$.

This force acts over the average cylindrical area 


$$
\bar{A}=2 \pi \bar{r} l_{\text {coil }}
$$

resulting in a magnetic pressure

$$
P_{B}=F_{r} / \bar{A} .
$$

The average magnetic and gas-dynamic pressure for each coil geometry is shown in Fig. 13 for three different radial positions of the current sheet. These radial positions correspond to displacement to $25 \%$, $50 \%$, and $75 \%$ of the initial average coil radius. In other words, this simulates when the current sheet average radius has compressed to $25 \%$ (most pinched case), $50 \%$, and $75 \%$ (least pinched case) of the average coil radius.
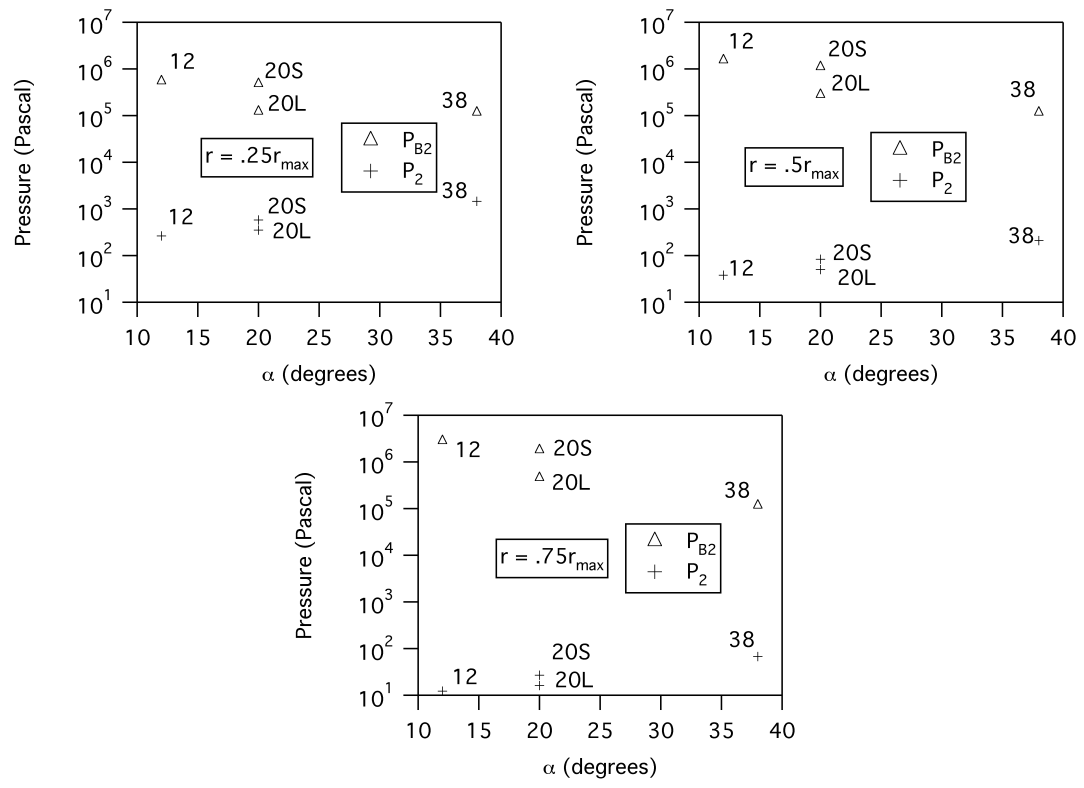

Figure 13. Results of magnetic and gas-dynamic pressure calculations at three different radial positions representing displacement from the coil to $25 \%, 50 \%$, and $75 \%$ of the average coil radius.

With the magnetic pressure orders of magnitude greater than $P_{2}$, we expect very minimal additional axial acceleration through gas-dynamic effects. It should be noted that these calculations are appropriate for conical theta pinch accelerators, and similar pinching devices that operate at mass flow rates on the order of $1 \mathrm{mg} / \mathrm{s}$ and have no imbedded magnetic field in the propellant prior to the main current discharge through the inductive coil. The pinching contribution to thrust is orders of magnitude lower than the electromagnetic contribution to thrust in the present case, but devices that operate at a higher mass flow rate or those that embed a magnetic field in the propellant could have a higher pressure associated with the volume bounded by the current sheet. Devices with these characteristics would reach an equilibrium condition through a combination of gas-dynamic and magnetic pressure both resisting radial compression of the propellant, reducing the overall pinching and attenuating inductive decoupling of the plasma from the driving coil.

\section{Conclusions}

We have presented results demonstrating the effect of coil geometry and inductive current sheet trajectory on the performance of a conical theta pinch pulsed inductive plasma accelerator. The inductive coupling between the coil of the thruster and the plasma current sheet as a function of plasma displacement was experimentally simulated for four different coil geometries. An empirical expression for the inductance presented to the thruster terminals as a function of current sheet axial position was fit to experimental data and used to close a system of coupled ODEs describing the acceleration of the plasma, which were numerically solved for the exhaust velocity as a function of time for two current sheet trajectories for each of 
four inductive coil geometries. Both trajectories were for purely axial displacement of the plasma, with the first plasma current sheet geometrically mirroring the inductive coil and the second radially pinched inward a constant amount throughout the acceleration process. The final axial exhaust velocity was $10-30 \%$ lower when the current sheet was pinched, with a decrease in final kinetic energy of 20-50\%. Additional radial compression is expected to further exacerbate these losses. As the coil and current sheet length is increased for a constant minimum radius $r_{\text {coil }}$, the calculated exhaust velocity increases more slowly, but for a longer period of time leading to greater overall acceleration with a difference in final velocity of roughly $10 \%$.

Physically, we demonstrated that as the plasma radially compresses it decouples from the inductive coil, expending some energy in electromagnetic pinching without later recovering the energy in axial acceleration of the plasma. This decoupling results in a smaller percentage of the inductive stroke length available for axial acceleration, reducing the overall achievable exhaust velocity. Larger cone angles attenuate the inductive decoupling between coil and current sheet in the event of radial compression, resulting in a larger fraction of the potential electromagnetic work directed into axial current sheet acceleration relative to the smaller cone angles. Estimates of the gasdynamic pressure in the pinched current sheet of accelerators like the MAD-IPA as compared to the magnetic pressure on the plasma imply a very low potential that the energy expended compressing the plasma can be recovered as axially-directed kinetic energy.

\section{Acknowledgments}

The authors appreciate the help and support of Dr. Bill Emrich, Dr. Adam Martin, Mr. Richard Eskridge, Mr. J. Boise Pearson, and Mr. Jim Martin, and thank Mr. Tommy Reid, Mr. Douglas Galloway, Mr. M. Keith Chavers, Mr. Adam Kimberlin, and Mr. Mark Becnel for their invaluable technical assistance. This work was supported in part by NASA's Advanced In Space Propulsion Program managed by Dr. Michael LaPointe.

\section{References}

${ }^{1}$ Polzin, K. A. Comprehensive review of planar pulsed inductive plasma thruster research and technology. Journal of Propulsion and Power, 27(3):513-531, May-June 2011.

${ }^{2}$ Lovberg, R. H. and Dailey, C. L. A PIT primer. Technical Report 005, RLD Associates, Encino, CA, 1994.

${ }^{3}$ Dailey, C. L. and Lovberg, R. H. The PIT MkV Pulsed Inductive Thruster. Technical Report 191155, Lewis Research Center, Redondo Beach, CA, July 1993.

${ }^{4}$ Polzin, K. A. Markusic, T. E. Stanojev, B. J. DeHoyos, A. and Spaun, B. Thrust stand for electric propulsion performance evaluation. Review of Scientific Instruments, 77:105108, October 2006.

${ }^{5}$ Polzin, K. A. Scaling and System Considerations in Pulsed Inductive Plasma Thrusters. IEEE Transactions on Plasma Science, 36(5):2189-2198, October 2008.

${ }^{6}$ Josephson, V. and Hales, R. W. Parametric Study of the conical Shock Tube. The Physics of Fluids, 4(3):373-379, 1961.

${ }^{7}$ Cassibry, J. T. Fimognary, P. J. and Ims, K. E. Effects of Pre-ionization and Bias Field on Plasmoid Formation and Acceleration. Number AIAA-2007-5262, July 2007.

${ }^{8}$ A. K. Martin M. H. Lee R. H. Eskridge, P. J. Fimognari. Design and construction of the PT-1 Prototype Plasmoid Thruster. Technical report, Propulsion Research Center, NASA Marshall Space Flight Center, 2005.

${ }^{9}$ Josephson, V. Production of High Velocity Shocks. Journal of Applied Physics, 29(1):30-32, 1958.

${ }^{10}$ Cruddace, R. C. and Hill, M. Mechanism of Plasma Acceleration in a Conical Theta-pinch Gun. Technical Report CLM-M52, Culham Laboratory, 1966.

${ }^{11}$ Niemela, C. S. and Kirtley, D. E. Initial Results on an Annular Field Reversed configuration Plasma Translation Experiment. Technical Report AFRL-RZ-ED-TP-2008-489, Air Force Research Laboratory, November 2008.

${ }^{12}$ Kirtley, D. E. Gallimore, A. D. Haas, J. and Reilly, M. High density magnetized toroid formation and translation with xocot: An annular field reversed configuration plasma concept. Technical Report AFRL-PR-ED-TP-2007-387, Air Force Research Laboratory, August 2007.

${ }^{13}$ Miller, R. Best, S Ownes, T. Polzin, K. A., Rose, F. M. and Dankanich, J. Design of a Low-Energy FARAD Thruster. Number AIAA 2007-5257, July 2007.

${ }^{14}$ Hallock, A. K. Polzin, K. A. and Gregory Emsellem. Design of a microwave assisted discharge inductive plasma accelerator. Number SP-2010-1842709, May 2010.

${ }^{15}$ Hallock, A. K. and Polzin, K. A. . Design of a microwave assisted discharge inductive plasma accelerator. Number AIAA-2010-6527, August 2010.

${ }^{16}$ Martin, A. K. and Eskridge, R. H. Electrical coupling efficiency of inductive plasma accelerators. Journal of Physics D: Applied Physics, 38, 2005.

${ }^{17}$ Bernardes, J. Merryman, S. Rose, M. F. and Martin, T. H. Parameter Analysis of a Single Stage Induction Mass Driver. Technical report, 5th IEEE Int. Pulsed Power conf. 